\title{
Recent Trends and Future Directions in Vertex-Transitive Graphs
}

\author{
Klavdija Kutnar * \\ University of Primorska, FAMNIT, Glagoljaška 8, 6000 Koper, Slovenia \\ Dragan Marušič * \\ University of Primorska, FAMNIT, Glagoljaška 8, 6000 Koper, Slovenia \\ University of Ljubljana, PEF, Kardeljeva ploščad 16, 1000 Ljubljana, Slovenia
}

Received 3 August 2008, accepted 19 August 2008, published online 22 August 2008

\begin{abstract}
A graph is said to be vertex-transitive if its automorphism group acts transitively on the vertex set. Some recent developments and possible future directions regarding two famous open problems, asking about existence of Hamilton paths and existence of semiregular automorphisms in vertex-transitive graphs, are discussed, together with some recent results on arc-transitive graphs and half-arc-transitive graphs, two special classes of vertex-transitive graphs that have received particular attention over the last decade.
\end{abstract}

Keywords: Vertex-transitive graph, arc-transitive graph, half-arc-transitive graph, Hamilton cycle, Hamilton path, semiregular group, (im)primitive group.

Math. Subj. Class.: 05C25, 20B25

\section{In the beginning}

Vertex-transitive graphs, that is, graphs whose automorphisms groups acts transitively on the corresponding vertex sets, have been an active topic of research for a long time now. Much of this interest is due to their suitability to model scientific phenomena when symmetry is an issue. It is the aim of this article to discuss recent developments surrounding two well known open problems in vertex-transitive graphs - the Hamilton path/cycle problem and the semiregularity problem - as well as the general question addressing structural properties of arc-transitive and half-arc-transitive graphs. In doing so we will also try to contemplate possible directions this area of research is likely to take in the near future.

\footnotetext{
*Supported by "Agencija za raziskovalno dejavnost Republike Slovenije", research program P1-0285.

E-mail addresses: klavdija.kutnar@upr.si (Klavdija Kutnar),dragan.marusic@upr.si (Dragan Marušič)
} 
The article is organized as follows. In Section 2 we discuss the problem, posed by the second author in 1981 (see [79]) who asked if it is true that a vertex-transitive digraph contains a nonidentity automorphism with all orbits of equal length, in short, a semiregular automorphism. Section 3 gives a quick overview of the problem, posed by Lovàsz in 1969 (see [72]), who asked if it is true that every connected vertex-transitive graph contains a Hamilton path, thus motivating a great deal of research into vertex-transitive graphs in the following decades. In Section 4, imprimitivity, one of the most fundamental concepts in the theory of permutation groups, is considered with special emphasis given to a recently developed theory which links the existence of blocks of imprimitivity in vertex-transitive graphs, having an abelian semiregular subgroup of automorphisms, to certain conditions that need to be satisfied in the corresponding quotient graph relative to the orbits of such a subgroup. Finally, in Section 5 we deal with some recent structural results on arc-transitive and half-arc-transitive graphs, as well as their link to some open problems in the theory of configurations.

For group-theoretic terms not defined here we refer the reader to [124].

\section{Semiregularity}

Let $G$ be a permutation group on a finite set $V$. A non-identity element of $G$ is semiregular, more precisely $(m, n)$ - semiregular, if it has $m$ orbits of size $n$. It is known that each finite transitive permutation group contains a fixed-point-free element of prime power order [43, Theorem 1], but not necessarily a fixed-point-free element of prime order and, hence, no semiregular element. A permutation group with no semiregular elements is sometimes called elusive (see [25]). The name is intended to suggest that such groups appear to be quite rare. Indeed, a first construction of elusive groups (associated with Mersenne primes) was described in [43]. More recently, infinite families of such groups were given in [25, 49, 50].

One would expect "nice" combinatorial objects, for example graphs, to have non-elusive automorphism groups. Indeed, the problem first arose in a graph-theoretic context in 1981 when it was asked if every vertex-transitive digraph has a semiregular automorphism [79, Problem 2.4]. There has recently been an increased interest in this problem, now know as the semiregularity problem, measured, among others, by a number of articles, manuscripts and other forms of written material. Mostly, these articles make small, but important, steps towards a possible final answer to the problem. In short, the problem is still open.

The now commonly accepted, and slightly more general, version of the semiregularity problem involves the whole class of 2-closed transitive groups [24, 62]. Following [125], the 2-closure $G^{(2)}$ of a permutation group $G$ is the largest subgroup of the symmetric group $S_{V}$ having the same orbits on $V \times V$ as $G$; alternatively, $G^{(2)}$ is the intersection of the automorphism groups of all orbital digraphs associated with the action of $G$ on $V$. The group $G$ is said to be 2 -closed if it coincides with $G^{(2)}$. We remark that the 2-closures of all elusive groups mentioned in $[25,49,50]$ are non-elusive, thus supporting the above conjecture.

Three lines of approach to the semiregularity problem may be identified: the order of a graph, the valency of a graph, and special types of action of the group. For instance, if $p$ is a prime then transitive permutation groups of degree $p^{k}, k \geq 1$, or $m p$, with $m \leq p$, contain semiregular elements [79]. Also, the automorphism groups of vertex-transitive digraphs on $2 p^{2}$ vertices, and by the most recent result, 2-closed groups of square-free degree are known to contain semiregular elements [38,97]. Further, cubic and quartic vertex-transitive graphs have semiregular automorphisms $[39,97]$. Perhaps the most important work on the subject is due to Giudici [49] who proved that, with the exception of a certain family of groups as- 
sociated with $M_{11}$, all quasiprimitive permutation groups contain semiregular elements, and even those which do not, have 2-closures which do. (A permutation group is quasiprimitive if every non-trivial normal subgroup is transitive.) Very recently, Giudici and $\mathrm{Xu}$ [51] extended these results by combining valency and type of action approaches to classify all biquasiprimitive groups without semiregular elements, and to prove the existence of semiregular automorphisms in vertex-transitive graphs whose vertex stabilizers act quasiprimitively on the corresponding sets of neighbors. (A biquasiprimitive permutation group is a transitive permutation group for which every nontrivial normal subgroup has at most two orbits and there is some normal subgroup with precisely two orbits.) As an important consequence, for example, arc-transitive graphs with prime valency, and 2-arc-transitive graphs have semiregular automorphisms. Further, $\mathrm{Xu}$ [128] has shown the existence of desired automorphisms whenever the graph is arc-transitive of valency a product of two primes as long as its automorphism group has a nonabelian minimal normal subgroup with at least three orbits on the vertex set.

These results, chipping away the nonsolvable side of the equation, suggest an inevitable shift of emphasis to solvable groups is needed, if one is to hope for a solution of the problem. Namely, in view of the above results on quasiprimitive and biquasiprimitive groups, one may restrict oneself to transitive 2-closed groups which have a "genuine" imprimitivity block system arising from an intransitive normal subgroup with at least three orbits. This allows a normal subgroup reduction to a smaller quotient graph which admits a vertex-transitive action of the quotient group. This would, at least in principle, set the stage for an induction type of argument (see Proposition 3). It seems reasonable to start out the research in the genuinely imprimitive case by first considering solvable groups, where the normal subgroup reduction has obvious specific advantages. In fact in this special case of the semiregularity problem for solvable groups we may always assume that there exists an genuine imprimitivity block system. Namely, let $G$ be a transitive solvable group and let $N=\mathbb{Z}_{p}^{m}$ be the minimal elementary abelian normal subgroup of $G$. If $N$ is transitive then all of its nontrivial elements are semiregular. If, on the other hand, $N$ has two orbits, say $O_{1}$ and $O_{2}$ then, by [38, Proposition 3.1], either the restriction of every element of order $p$ is semiregular on both $O_{1}$ and $\mathrm{O}_{2}$, or there exist an element which has prime order on $\mathrm{O}_{1}$ and is the identity on $\mathrm{O}_{2}$, and an element which has prime order on $O_{2}$ and is the identity on $O_{1}$. But then the product of these two elements is semiregular. We therefore pose the following two problems, the second being a special case of the first one.

Problem 1. Does a vertex-transitive graph with genuinely imprimitve automorphism group contain a semiregular element?

Problem 2. Does a vertex-transitive graph with solvable automorphism group contain a semiregular element?

The above mentioned normal subgroup reduction approach gives an affirmative answer in the special case of Problem 2 where the graphs in questions admit a transitive group of automorphisms all of whose Sylow subgroups are cyclic.

Proposition 3. Let $X$ be a vertex-transitive graph such that all Sylow subgroups of its automorphism group Aut $X$ are cyclic. Then $X$ has a semiregular automorphism.

Proof. The classical result of Burnside [23] says that a group $H$ with a cyclic Sylow $p$ subgroup $P$, where $p$ is the smallest prime divisor of $|H|$, is a product of the form $K P$, where $K$ is a normal subgroup (of order prime to $p$ ). 
Let now $G \leq$ Aut $X$ be an arbitrary transitive subgroup and let $p$ be the smallest prime divisor of $|G|$. Then, by the above result of Burnside we have that $G=P K$ where $K$ is a normal subgroup in $G$ of order prime to $p$. If $K$ acts intransitively on $X$ then the quotient group $G / K$ acts transitively on the quotient graph $X_{K}$ relative to the orbits of $K$. (The vertex set of $X_{K}$ coincides with the set of orbits of $K$ with the edge set induced naturally by the edge set of $X$.) Since $G / K$ is isomorphic to a Sylow $p$-subgroup $P$, by standard orbit-stabilizer property, the quotient graph $X_{K}$ is of prime power order $p^{i}$, for some $i \in \mathbb{N}$, and hence, as mentioned in one of the preceding paragraphs, by [79], $G / K$ contains a semiregular element $\alpha$ of order $p$. Since $|K|$ is of order coprime to $p$ it follows that the lift of $\alpha$ in $G$ is a semiregular automorphism of $X$. If however $K$ acts transitively on $X$, then take the smallest prime divisor of $|K|$ and repeat the argument. Either along this process the existence of a semiregular automorphism is assured or a Sylow $q$-subgroup $Q$, where $q$ is the biggest prime dividing $|G|$ acts transitively on $X$, and hence $X$ clearly has a semiregular automorphism.

\section{Hamilton paths and cycles}

The solution of the semiregularity problem would certainly contribute significantly to various open problems regarding vertex-transitive graphs. One of such problems is the problem of existence of Hamilton paths/cycles (that is, a simple paths/cycles going through all vertices) in finite connected vertex-transitive graphs. In particular, in 1969 Lovász [72] asked whether every finite connected vertex-transitive graph has a Hamilton path. All known connected vertex-transitive graphs have a Hamilton path and with the exception of $K_{2}$, only four connected vertex-transitive graphs that do not have a Hamilton cycle are known to exist: the Petersen graph, the Coxeter graph and the two graphs obtained from them by replacing each vertex by a triangle. The fact that none of these four graphs is a Cayley graph, that is, a vertextransitive graph with a regular subgroup of automorphisms, has led to a folklore conjecture that every connected Cayley graph has a Hamilton cycle.

This problem, together with its Cayley graph variation, produced conjectures and counterconjectures with regards to its truthfulness. For example, Thomassen's conjecture [15, 122] says that only finitely many connected vertex-transitive graphs without a Hamilton cycle exist, whereas Babai's conjecture $[12,13]$ says that infinitely many such graphs exist. A large number of articles directly or indirectly related to this problem (for the list of relevant references and a detailed description of the status of this problem see [67]), have appeared in the literature, affirming the existence of such paths in some special vertex-transitive graph and, in some cases, also the existence of Hamilton cycles. It is known that connected vertex-transitive graphs of order $k p$, where $k \leq 4$, and $p^{j}$, where $j \leq 4$, and $2 p^{2}$, where $p$ is a prime, (except for the Petersen graph and the Coxeter graph) contain a Hamilton cycle, whereas for connected vertex-transitive graphs of order $5 p$ and $6 p$ it is known that they contain Hamilton paths (see $[1,2,3,4,5,6,8,9,10,11,26,65,68,81,82,83,89,90,119])$. Particular attention has been given to Cayley graphs. For example, one may easily see that connected Cayley graphs of abelian groups have a Hamilton cycle. Further, following $[42,61,80]$ it is now known, that with the exception of the Petersen graph every connected vertex-transitive graph whose automorphism group contain a transitive subgroup with the cyclic commutator subgroup of prime power order, has a Hamilton cycle [37]. Of many positive results for Cayley graphs arising from particular generating sets it is worth mentioning the most recent result of this kind. It was recently shown in [52], innovatively combining algebraical and topological tools, that cubic Cayley graphs arising from finite groups $G=\left\langle a, x \mid a^{2}=1, x^{s}=1,(a x)^{3}=1, \ldots\right\rangle$ 
having a $(2, s, 3)$-presentation contain a Hamilton cycle when $|G|$ is congruent to 2 modulo 4 , and contain a Hamilton path when $|G|$ is congruent to 0 modulo 4 .

A frequently used approach to constructing Hamilton cycles in vertex-transitive graphs is based on a quotienting with respect to a suitable semiregular automorphism or with respect to an imprimitivity block system of the corresponding automorphism group. If the quotient graph contains a Hamilton cycle then it is sometimes possible to lift this cycle - spiraling through the corresponding blocks/orbits - to a Hamilton cycle in the original graph. It can be easily seen that such lifts of Hamilton cycles from quotient graphs are always possible, for example, when the quotienting is done relative to a semiregular automorphism of prime order and where in the quotient graph there are at least two adjacent orbits joined by a double edge. This suggests that the question that should be addressed in the context of lifts of Hamilton cycles concerns the case when any two adjacent orbits in the quotient graph are joined by a single edge. In other words, when is a covering graph of a vertex-transitive graph containing a Hamilton cycle also hamiltonian? For example, it is fairly easy to see that the remarkable result of Witte about Hamilton cycles in Cayley (di)graphs of $p$-groups [127] could be successfully generalized to arbitrary vertex-transitive graphs of prime power order - which is in our opinion perhaps the most important next step needed to be taken if one is to obtain a complete solution to the Hamilton path/cycle problem - provided one could prove that for a prime $p$, a connected regular $\mathbb{Z}_{p}$-cover of a hamiltonian vertex-transitive graph of order a power of $p$, is hamiltonian.

\section{Imprimitivity}

A permutation group $G$, acting transitively on a set $X$, is imprimitive if there exists a nontrivial equivalence relation $R$ on $X$ with its equivalence classes invariant under the action of $G$. Such a relation is called $G$-invariant and its equivalence classes are called blocks of imprimitivity. For example, $G$ is imprimitive whenever it contains a nontrivial intransitive normal subgroup, since the orbits of a normal subgroup are always $G$-invariant. As mentioned in Section 2, imprimitive actions where no nontrivial $G$-invariant relation arises in such a way are called quasiprimitive. A transitive permutation group is primitive if it is not imprimitive.

Imprimitivity is one of the most fundamental concepts in the theory of permutation groups, primarily because the action of $G$ on $X$ may be recaptured, at least to some extent, from the induced transitive action of $G$ on a smaller quotient set $X / R$. By repeating this process we eventually end up with primitive actions. To take advantage of this idea, one needs an appropriate description of a large enough class of invariant relations in question. A class of actions where this approach proves to be promising is the case when $G$ contains a semiregular abelian subgroup $H$. (We note that asking for $G$ to contain such a subgroup is not that restrictive, in view of the conjecture given in Section 2.) In this case, all $G$-invariant relations admit a reasonably simple description in terms of orbits of certain subgroups of $H$. In particular, there is an interesting subclass of relations obtained as follows. Let $X$ be an auxiliary digraph with the vertex set $V$ which admits $G$ as a group of automorphisms, let $W$ be an eigenspace of $X$, and let $N$ be the kernel of the linear representation of $G$ on $W$. Then we define $R$ to be the $G$-invariant equivalence relation arising from the orbits of $N$. Recent work on this subject [64] has shown that relations arising in such a way admit a particularly detailed description. Future directions in the study of imprimitivity block systems are likely to use this approach.

As mentioned in [64], in a graph-theoretic setting, problems of this kind are motivated by the following situation, often encountered when dealing with questions regarding symmetry 
of graphs. Given a graph $X$ admitting a cyclic subgroup with two orbits of equal size, there are two essentially different possibilities forcing such a graph to be vertex-transitive. Either there exists an automorphism swapping setwise the two orbits, or there exists an automorphism not swapping the two orbits while sending at least one vertex of the first orbit to a vertex in the second orbit. Of course, the existence of an automorphism of the first kind in $X$ is equivalent to there being a transitive subgroup of automorphisms with the two orbits of the cyclic group in question as blocks of imprimitivity. On the other hand, the existence of an automorphism of the second kind in $X$ means that the two orbits of the semiregular cyclic subgroup do not form blocks of imprimitivity for the full automorphism group. For example, it is easy to see that the class of generalized Petersen graphs [48] contains examples of each of the four possibilities that may occur with this respect. It seems therefore natural to seek for (non)existence conditions for these two kinds of automorphisms in $X$.

Problem 4. Given a vertex-transitive graph $X$ with a $(2, n)$-semiregular automorphism $\alpha$, find necessary and sufficient conditions for the existence of an automorphism swapping the two orbits of $\alpha$, and find necessary and sufficient conditions for the existence of an automorphism not swapping the two orbits of $\alpha$ while sending at least one vertex of the first orbit to a vertex in the second orbit.

More generally, let us consider all connected transitive graphs $X$ arising, up to isomorphism of covering projections, as regular abelian covers $p: X \rightarrow Y$ of a fixed given (possibly nonsimple or with semi-edges) graph $Y$. (For graph-covering terms not defined here we refer the reader to [55, 102, 105].) As shown by Djoković [35], symmetry properties of $X$ are, to some extent, reflected by the symmetries of $Y$ provided that enough automorphisms lift along $p$. The lifting problem is well understood, see [35, 40, 44, 73, 76, 77, 78]. Thus, studying symmetries of $X$ arising via lifting automorphisms should be considered 'easy'. It may happen, though, that not all symmetries of $X$ arise this way. In particular, $X$ could be transitive, even if $Y$ is not. So, what one wants is to decompose the covering projection as $p=q r$ in such a way that all symmetries of $X$ arise via lifting automorphisms of an intermediate graph $Y^{\prime}$ along $r: X \rightarrow Y^{\prime}$. This is achieved by taking $r$ to be the regular quotient by a normal subgroup $N$ of Aut $X$ which is contained in the group of covering transformations $\operatorname{CT}(p)$. Repeating the reduction one eventually come to a, hopefully, small set of 'basic graphs' out of which all graphs $X$ arise 'nicely'. Of course, what one should ask of these 'basic graphs', in the first place, is that there should be a reasonably easy way of finding them. This imposes certain restrictions as to how the reduction is done - making an appropriate choice of $N$ above - and here the idea of taking $N$ to be the kernel of the representation of the action of Aut $X$ on an eigenspace of $X$ comes into play. The 'basic graphs' are then characterized as those which cannot be decomposed further in this manner, see $[63,64]$ for a characterization of a class of arc-transitive cyclic covers and a class of bi-Cayley graphs on abelian groups. (A bi-Cayley graph $X$ is a graph which admits a semiregular group of automorphisms $G$ with two orbits. Some authors use the term semi-Cayley instead $[69,111]$.) In short, we pose the following problem.

Problem 5. Given a connected graph $Y$ on a reasonably small number of vertices, classify, up to isomorphism of covering projections, all connected arc-transitive abelian covers of $Y$.

Observe that the above problem is related to the semiregularity problem from Section 2. Namely, a graph contains a $(m, n)$-semiregular automorphism if and only if it is a $\mathbb{Z}_{n}$-cover of a graph on $m$-vertices. 
Problem 5 is solved in [63] for a particular graph on two vertices. Namely let $Y$ be a graph of order 2 with a single edge between the two vertices and possibly some loops at each of the two vertices. (A special case of such graphs are the generalized Petersen graphs [48].) Then a cover of $Y$ is a bi-Cayley graph, in particular, using the terminology of [63], a onematching bi-Cayley graph. We would like to mention that the classification of one-matching bi-Cayley graphs done in [63] is "classification of finite simple groups free", which is usually not the case in solutions to problems of this kind.

\section{Structural properties}

In 1947 Tutte [120] showed that the maximum $s$-arc-transitivity of a cubic graph is 5 , thus opening up a very fruitful area of research. Later on Djoković and Miller [36] proved that a vertex stabilizer in an $s$-regular subgroup of automorphisms of a cubic arc-transitive graph is isomorphic to $\mathbb{Z}_{3}, S_{3}, S_{3} \times \mathbb{Z}_{2}, S_{4}$, or $S_{4} \times \mathbb{Z}_{2}$ depending on whether $s=1,2,3,4$ or 5 , respectively. In 1981, using the classification of finite simple groups, Weiss [126] generalized Tutte's result by showing that for an $s$-arc-transitive graph of valency greater than 2 we must necessarily have that $s \in\{1,2,3,4,5,7\}$.

Not surprisingly arc-transitive graphs, and cubic arc-transitive graphs in particular, have received considerable attention over the years, the aim being to obtain structural results and possibly a classification of such graphs of different transitivity degrees, particular orders or satisfying additional properties (see, for example [27, 30, 31, 44, 45, 46, 47, 60, 70, 106, 107, $108,109,110,130])$. The frequently used methods in this respect are based on covering graph techniques while using a particular additional condition about their automorphism groups such as, for example, imprimitivity or existence of particular semiregular automorphisms (see Sections 2 and 4). Also, the concept of consistent cycles together with a beautiful result of Conway $[17,32]$ which says that given an arc-transitive graph $X$ of valency $d$, an arctransitive subgroup $G$ of Aut $X$ has $d-1$ orbits on $G$-consistent cycles, is, for example, an essential part of the approach lead to the complete classification of cubic arc-transitive graphs to girth 6 , see [66]. (A walk $D=\left(u_{0}, \ldots, u_{r}\right)$ in a graph $X$ is called $G$-consistent, where $G \leq$ Aut $X$, if there exists $g \in G$ such that $u_{i}^{g}=u_{i+1}$ for $i \in\{0,1, \ldots, r-1\}$.) We believe that similar applications of Conway's result as well as its recent generalizations due to Miklavič, Potočnik and Wilson [100, 101] will prove useful in future investigations of symmetry properties in graphs.

Another interesting area of research are the so called half-arc-transitive graphs. Clearly, a graph that is arc-transitive is also vertex-transitive and edge-transitive. But the converse is not true in general. In particular, in 1966 Tutte [121] proved that the automorphism group of a vertex-transitive and edge-transitive graph which is not arc-transitive has two orbits of equal size on the arc set, and consequently that a vertex-transitive and edge-transitive graph of odd valency is arc-transitive. A graph that is vertex-transitive and edge-transitive but not arc-transitive is called half-arc-transitive graph. In 1966 Tutte [121] asked if for every given even integer $k \geq 4$ there exists a half-arc-transitive graph of valency $k$. A positive answer was given by Bouwer in [21] by a construction of a half-arc-transitive graph of valency $k$ for any even integer $k \geq 4$. The smallest known example of a half-arc-transitive graph is the Doyle-Holt graph [7, 59] which is quartic and of order 27. Graphs admitting a halfarc-transitive group actions are in a one-to-one correspondence with the so called orbital graphs of groups with non-self-paired orbitals whereas graphs admitting arc-transitive group actions are in a one-to-one correspondence with orbital graphs of groups with self-paired 
orbitals. In particular, let $G$ be a transitive permutation group acting on a set $V$, and let $\mathcal{O} \neq\{(v, v) \mid v \in V\}$ be a nontrivial orbital in the natural action of $G$ on $V \times V$. Then the group $G$ acts half-arc-transitively on the corresponding orbital graph with vertex set $V$ and edge set $\{u v \mid(u, v) \in \mathcal{O}\}$ when $\mathcal{O} \neq \mathcal{O}^{*}$ and it acts arc-transitively when $\mathcal{O}=\mathcal{O}^{*}$, where $\mathcal{O}^{*}=\{(u, v) \mid(v, u) \in \mathcal{O}\}$ is the paired orbital of $\mathcal{O}$.

Half-arc-transitive graphs, quartic half-arc-transitive graphs in particular, and graphs admitting half-arc-transitive group actions in general have been an active topic of research in the last decades. In particular, a classification of certain restricted families and various constructions of new families of such graphs together with some structural properties are known, see $[11,29,41,71,74,75,84,85,86,87,88,98,99,112,115,116,117$, $118,123,129]$. There are several approaches used in this respect, such as for example, investigation of the (im)primitivity of half-arc-transitive group actions on graphs, geometry related questions, and questions concerning classification for various restricted families of half-arc-transitive graphs. In this context, further structural results about quartic halfarc-transitive graphs are most sought for. The concept of alter-exponent in digraphs, introduced in [95], turns out to be useful in this respect. In particular, given a digraph $D$, $\left\{v_{0}, v_{1}, \ldots, v_{n}\right\} \subseteq V(D)$ and $\left\{a_{1}, a_{2}, \ldots, a_{n}\right\} \subseteq A(D)$ is a subset of the arc set of $D$, a sequence $W=\left(v_{0}, a_{1}, v_{1}, a_{2}, v_{2}, \ldots, v_{n-1}, a_{n}, v_{n}\right)$ is a walk of length $n$ in $D$ from $v_{0}$ to $v_{n}$ if for all $i \in\{1,2, \ldots, n\}$ either $a_{i}=\left(v_{i-1}, v_{i}\right)$ or $a_{i}=\left(v_{i}, v_{i-1}\right)$. In the first case $a_{i}$ is positively oriented in $W$, and is negatively oriented in the second case. The sum $s(W)$ of the walk $W$ is the difference between the number of positively oriented arcs in the walk and the number of negatively oriented arcs in the walk. The tolerance of $W$ is the set $\left\{s_{k}(W) \mid k \in\{0,1, \ldots, n\}\right\}$, where $s_{k}(W)$ is the sum of the subwalk of $W$ from $v_{0}$ to $v_{k}$ and $s_{0}(W)=0$. Observe that the tolerance of a walk is always an interval of integers containing 0 . We say that two vertices $u$ and $v$ of a digraph $D$ are alter-equivalent with tolerance $I$ if there is a walk from $u$ to $v$ with sum 0 and tolerance $J, J \subseteq I$. It is not difficult to see that this relation is an equivalence relation. Let the corresponding partition of $V(D)$ be denoted by $B_{I}(D)$. Then the alter-exponent of a digraph $D$ is the smallest positive integer $t$, for which $B_{[0, t]}(D)=B_{[0, \infty]}(D)$. Clearly, each graph $X$ admitting a half-arc-transitive action of a group $G$ with respect to the non-self-paired orbital $\mathcal{O}$ can be viewed as a vertex-transitive digraph $D$ with vertex set $V(D)=V(X)$ and $\operatorname{arc} \operatorname{set} A(D)=\mathcal{O}$, and thus the concept of alter-exponent, in particular $G$-alter-exponent, can be applied. Quartic graphs admitting a half-arc-transitive action with respect to which the corresponding alter-exponent equals 1 are called tightly attached graphs and they has been completely classified (see [84, 96, 115]). Thus a natural next step in this direction would be to solve the following problem posed in [95].

Problem 6. [95] Classify quartic graphs admitting a half-arc-transitive action with respect to which the corresponding alter-exponent equals 2 .

Finally, let us mention that vertex-transitive graphs have many applications, sometimes in quite surprising ways. One of such applications concerns configurations. Structural results on vertex-transitive graphs are useful, for example, in the context of symmetric configurations because there is a one-to-one correspondence between bipartite vertex-transitive graphs of girth at least 6 and the Levi graphs of self-dual, point- and line-transitive combinatorial configurations. Similarly, bipartite arc-transitive graphs of girth at least 6 are in a one-to-one correspondence with the Levi graphs of flag-transitive combinatorial configurations, and bipartite half-arc-transitive graphs of girth at least 6 are in a one-to-one correspondence with 
the Levi graphs of weakly flag-transitive combinatorial configurations. (A symmetric combinatorial $\left(v_{r}\right)$ configuration consists of $v$ points and $v$ lines such that each point is incident to $r$ lines and each line is incident to $r$ points, and any pair of points is on at most one line and any pair of lines intersect in at most one point. A Levi graph of a configuration is a graph whose vertex set consists of points and lines of the configuration with the edge set mirroring the point/line incidence relation. A flag is a pair of incident point and line. A weakly flag-transitive configuration is a configuration whose group of automorphisms acts intransitively on flags but the group of all automorphisms and anti-automorphisms acts transitively on flags.)

Several results making use of this correspondence are known (see [28, 33, 58, 96, 92, $102])$. In this sense many open problems in symmetric configurations, such as, for example, open problems on self-dual, point- and line-transitive $\left(v_{3}\right)$ configurations is, through the above mentioned correspondence, are special cases of open problems on cubic vertextransitive graphs (see [19, 20, 22, 34, 58, 92, 94, 103]). And similarly, open problems concerning weakly flag-transitive configurations are special cases of open problems on halfarc-transitive graphs (see $[16,96,93])$. For further directions concerning configurations see $[14,18,33,53,54,56,57,113,114]$.

\section{Acknowledgment}

The authors wish to thank Aleksander Malnič and Štefko Miklavič, and the referees for helpful suggestions about the material in this article.

\section{References}

[1] B. Alspach, Hamiltonian cycles in vertex-transitive graphs of order $2 p$, Proceedings of the Tenth Southeastern Conference on Combinatorics, Graph Theory and Computing (Florida Atlantic Univ., Boca Raton, Fla., 1979), pp. 131-139, Congress. Numer, XXIII-XX, Utilitas Math., Winnipeg, Man., 1979.

[2] B. Alspach, Hamilton cycles in metacirculant graphs with prime power cardinal blocks, Graph theory in memory of G. A. Dirac (Sandbjerg, 1985), 7-16, Ann. Discrete Math. 41, North-Holland, Amsterdam, 1989.

[3] B. Alspach, Lifting Hamilton cycles of quotient graphs, Discrete Math. 78 (1989), 25-36.

[4] B. Alspach, C. C. Chen and K. McAvaney, On a class of Hamiltonian laceable 3-regular graphs, Discrete Math. 151 (1996), 19-38.

[5] B. Alspach, E. Durnberger and T. D. Parsons Hamilton cycles in metacirculant graphs with prime cardinality blocks, Cycles in graphs (Burnaby, B.C., 1982), 27-34, Ann. Discrete Math. 27, North-Holland, Amsterdam, 1985.

[6] B. Alspach, S. Locke and D. Witte, The Hamilton spaces of Cayley graphs on abelian groups, Discrete Math. 82 (1990), 113-126.

[7] B. Alspach, D. Marušič and L. Nowitz, Constructing graphs which are 1/2-transitive, J. Austral. Math. Soc. A 56 (1994), 391-402.

[8] B. Alspach and T. D. Parsons, On Hamiltonian cycles in metacirculant graphs, Algebraic and geometric combinatorics, 1-7, Ann. Discrete Math. 15 (1982), North-Holland, Amsterdam, 1982.

[9] B. Alspach and Y. S. Qin, Hamilton-connected Cayley graphs on Hamiltonian groups, European J. Combin. 22 (2001), 777-787.

[10] B. Alspach and and C. Q. Zhang, Hamilton cycles in cubic Cayley graphs on dihedral groups, Ars Combin. 28 (1989), 101-108.

[11] B. Alspach and M. Y. Xu, 1/2-arc-transitive graphs of order 3p, J. Alg. Combin. 3 (1994), 347-355.

[12] L. Babai, Problem 17, Unsolved Problems, Summer Research Workshop in Algebraic Combinatorics, Simon Fraser University, July, 1979. 
[13] L. Babai, Automorphism Groups, Isomorphism, Reconstruction, Chapter 27 of the Handbook of Combinatorics, 1447-1540, Edited by R. L. Graham, M. Grotschel and L. Lovász, North-Holland, 1995.

[14] L. W. Berman, A characterization of astral $\left(n_{4}\right)$ configurations, Discrete Comput. Geom. 26 (2001), 603612.

[15] J. -C. Bermond, Hamiltonian graphs, Selected Topics in Graph Theory, 127-167, Edited by L. W. Beinke and R. J. Wilson, Academic Press, London, 1978.

[16] A. Betten, G. Brinkmann, T. Pisanski, Counting symmetric configurations v $v_{3}$, Discrete Appl. Math. 99 (2000), $331-338$.

[17] N. Biggs, Aspects of symmetry in graphs, Algebraic methods in graph theory Vol. I, II (Szeged, 1978), pp. 27-35, Colloq. Math. Soc. János Bolyai 25 North-Holland, Amsterdam-New York, 1981.

[18] M. Boben, T. Pisanski, Polycyclic configurations, European J. Combin. 24 (2003), 431-457.

[19] M. Boben, T. Pisanski, and A. Žitnik, I-graphs, their Kronceker covers and corresponding configurations, submitted.

[20] M. Boben, T. Pisanski, A. Žitnik, and B. Grünbaum, Small triangle-free configurations of points and lines, submitted.

[21] I. Z. Bouwer, Vertex and edge-transitive but not 1-transitive graphs, Canad. Math. Bull. 13 (1970), 231-237.

[22] I. Z. Bouwer, W. W. Chernoff, B. Monson, Z. Star, The Foster Census, Charles Babbage Research Centre, 1988.

[23] W. Burnside, Notes on the theory of groups of finite order, Proc. London Math. Soc. 26 (1895), 191-214.

[24] P. J. Cameron (ed.), Problems from the Fifteenth British Combinatorial Conference, Discrete Math. 167/168 (1997), 605-615.

[25] P. J. Cameron, M. Giudici, W. M. Kantor, G. A. Jones, M. H. Klin, D. Marušič and L. A. Nowitz, Transitive permutation groups without semiregular subgroups J. London Math. Soc. 66 (2002), 325-333.

[26] Y. Q. Chen, On Hamiltonicity of vertex-transitive graphs and digraphs of order $p^{4}, J$. Combin. Theory Ser. B 72 (1998), 110-121.

[27] M. D. E. Conder and P. J. Lorimer, Automorphism groups of symmetric graphs of valency 3, J. Combin. Theory Ser. B 47 (1989), 60-72.

[28] M. Conder, A. Malnič, D. Marušič, T. Pisanski and P. Potočnik, The edge- but not vertex-transitive cubic graph on 112 vertices, J. Graph Theory 50 (2005), 25-42.

[29] M. D. E. Conder and D. Marušič, A tetravalent half-arc-transitive graph with nonabelian vertex stabilizer, $J$. Combin. Theory Ser. B $\mathbf{8 8}$ (2003), 67-76.

[30] M. D. E. Conder and M. Morton, Classification of trivalent symmetric graphs of small order, Australas. J. Combin. 11 (1995), 139-149.

[31] M. D. E. Conder and R. Nedela, Symmetric cubic graphs of small girth, J. Combin. Theory Ser. B 97 (2007), 757-768.

[32] J. H. Conway, Talk given at the Second British Combinatorial Conference at Royal Holloway College, 1971.

[33] H.S.M. Coxeter, Self-dual configurations and regular graphs, Bull. Amer. Math. Soc. 56 (1950), 413-455.

[34] H. S. M. Coxeter, R. Frucht, D. Powers, Zero-symmetric graphs. Academic Press, 1981.

[35] D. Ž. Djoković, Automorphisms of graphs and coverings, J. Combin. Theory Ser. B 16 (1974), $243-247$.

[36] D. Ž. Djoković and G. L. Miller, Regular groups of automorphisms of cubic graphs, J. Combin. Theory Ser. B 29 (1980), 195-230.

[37] E. Dobson, H. Gavlas, J. Morris and D. Witte, Automorphism groups with cyclic commutator subgroup and Hamilton cycles, Discrete Math. 189 (1998), 69-78.

[38] E. Dobson, A. Malnič, D. Marušič and L. A. Nowitz, Minimal normal subgroups of transitive permutation groups of square-free degree, Discrete Math. 307 (2007), 373-385.

[39] E. Dobson, A. Malnič, D. Marušič and L. A. Nowitz, Semiregular automorphisms of vertex-transitive graphs of certain valencies. J. Combin. Theory Ser. B 97 (2007), 371-380.

[40] S. F. Du, J. H. Kwak and M. Y. Xu, On 2-arc transitive covers of complete graphs with covering transformation group $\mathbb{Z}_{p}^{3}$, J. Combin. Theory Ser. B 93 (2005), 73-93. 
[41] S. F. Du and M. Y. Xu, Vertex-primitive 1/2-arc-transitive graphs of smallest order, Comm. Algebra 27 (1998), 163-171.

[42] E. Durnberger, Connected Cayley graphs of semi-direct products of cyclic groups of prime order by abelian groups are hamiltonian, Discrete Math. 46 (1983), 55-68.

[43] B. Fein, W. M. Kantor and M. Schacher, Relative Brauer groups II, J. Reine Angew. Mat. 328 (1981), 39-57.

[44] Y. Q. Feng and J. H. Kwak, One-regular cubic graphs of order a small number times a prime or a prime square, J. Austral. Math. Soc. 76 (2004), 345-356.

[45] Y. Q. Feng and J. H. Kwak, An infinite family of cubic one-regular graphs with unsolvable automorphism groups, Discrete Math. 269 (2003), 281-286.

[46] Y. Q. Feng and J. H. Kwak, Cubic symmetric graphs of order a small number times a prime or a prime square, J. Combin. Theory Ser. B 97 (2007), 627-646.

[47] Y. Q. Feng and K. Wang, s-Regular cyclic coverings of the three-dimensional hypercube $Q_{3}$, European J. Combin. 24 (2003), 719-731.

[48] R. Frucht, J. E. Graver and M. E. Watkins, The groups of the generalized Petersen graphs, Proc. Cambridge Philos. Soc. 70 (1971), 211-218.

[49] M. Giudici, Quasiprimitive groups with no fixed point free elements of prime order, J. London Math. Soc. 67 (2003), 73-84.

[50] M. Giudici, New constructions of groups without semiregular subgroups, Comm. Algebra 35 (2007), 27192730.

[51] M. Giudici and J. Xu, All vertex-transitive locally-quasiprimitive graphs have a semiregular automorphism, J. Algebr. Combin. 25 (2007), 217-232.

[52] H. H. Glover and D. Marušič, Hamiltonicity of cubic Cayley graph, J. Eur. Math. Soc. 9 (2007), 775-787.

[53] H. Gropp, Configurations and graphs, Discrete Math. 111 (1993), 269-276.

[54] H. Gropp, Configurations and graphs - II, Discrete Math. 164 (1997), 155-163.

[55] J. L. Gross and T. W. Tucker, “Topological Graph Theory”, Wiley - Interscience, New York, 1987.

[56] B. Grünbaum, Astral ( $\left.n_{k}\right)$ configurations, Geombinatorics 3 (1993), 32-37.

[57] B. Grünbaum, Configurations of points and lines, AMS, to appear.

[58] M. Hladnik, D. Marusic and T. Pisanski, Cyclic Haar graphs, Discrete Math. 244 (2002), 137-152.

[59] D. F. Holt, A graph which is edge transitive but not arc transitive, J. Graph Theory 5 (1981), 201-204.

[60] M. A. Iranmanesh, C. E. Praeger and S. Zhou, Finite symmetric graphs with two-arc transitive quotients, J. Combin. Theory Ser. B 94 (2005), 79-99.

[61] K. Keating and D. Witte, On Hamilton cycles in Cayley graphs in groups with cyclic commutator subgroup. Cycles in graphs (Burnaby, B.C., 1982), 89-102, Ann. Discrete Math. 27, North-Holland, Amsterdam, 1985.

[62] M. Klin, personal communication.

[63] I. Kovacs, A. Malnič, D. Marušič, Š. Miklavič, One-matching bi-Cayley graphs over abelian groups, European J. Combin., doi:10.1016/j.ejc.2008.06.001, in press.

[64] I. Kovacs, A. Malnič, D. Marušič, Š. Miklavič, Transitive group actions: (im)primitivity and semiregular subgroups, manuscript.

[65] K. Kutnar and D. Marušič, Hamiltonicity of vertex-transitive graphs of order $4 p$, European J. Combin. 29 (2008), 423-438.

[66] K. Kutnar and D. Marušič, A complete classification of cubic symmetric graphs of girth 6, J. Combin. Theory Ser. B, doi:10.1016/j.jctb.2008.06.001, in press.

[67] K. Kutnar and D. Marušič, Hamilton cycles and paths in vertex-transitive graphs - future directions, Discrete Math., submitted.

[68] K. Kutnar and P. Šparl, Hamiltonicity of vertex-transitive graphs of order $6 p$, submitted.

[69] K. H. Leung and S. L. Ma, Partial difference triples, J. Algebr. Combin. 2 (1993), 397-409.

[70] C. H. Li, Finite $s$-arc transitive graphs of prime-power order, Bull. London Math. Soc. 33 (2001), 129-137. 
[71] C. H. Li and H. S. Sims, On half-transitive metacirculant graphs of prime-power order, J. Combin. Theory Ser. B 81 (2001), 45-57.

[72] L. Lovász, "Combinatorial structures and their applications", (Proc. Calgary Internat. Conf., Calgary, Alberta, 1969), pp. 243-246, Problem 11, Gordon and Breach, New York, 1970.

[73] A. Malnič, Group actions, coverings and lifts of automorphisms, Discrete Math. 182 (1998), 203-218.

[74] A. Malnič and D. Marušič, Constructing 4-valent 1/2-transitive graphs with a nonsolvable group, J. Combin. Theory Ser. B 75 (1999), 46-55.

[75] A. Malnič and D. Marušič, Constructing 1/2-transitive graphs of valency 4 and vertex stabilizer $\mathbb{Z}_{2} \times \mathbb{Z}_{2}$, Discrete Math. 245 (2002), 203-216.

[76] A. Malnič, D. Marušič and P. Potočnik, Elementary abelian covers of graphs, J. Algebraic Combin. 20 (2004), 71-97.

[77] A. Malnič, D. Marušič, and P. Potočnik, On cubic graphs admitting an edge-transitive solvable group, J. Algebraic Combin. 20 (2004), 99-113.

[78] A. Malnič, R. Nedela and M. Škoviera, Lifting graph automorphisms by voltage assignments, European J. Combin. 21 (2000), 927-947.

[79] D. Marušič, On vertex symmetric digraphs, Discrete Math. 36 (1981), 69-81.

[80] D. Marušič, Hamilonian circuits in Cayley graphs, Discrete Math. 46 (1983), 49-54.

[81] D. Marušič, Vertex transitive graphs and digraphs of order $p^{k}$. Cycles in graphs (Burnaby, B.C., 1982), 115128, Ann. Discrete Math. 27, North-Holland, Amsterdam, 1985.

[82] D. Marušič, Hamiltonian cycles in vertex symmetric graphs of order $2 p^{2}$, Discrete Math. 66 (1987), $169-174$.

[83] D. Marušič, On vertex-transitive graphs of order qp, J. Combin. Math. Combin. Comput. 4 (1988), 97-114.

[84] D. Marušič, Half-transitive group actions on finite graphs of valency 4, J. Combin. Theory Ser. B 73 (1998), 41-76.

[85] D. Marušič, Quartic half-arc-transitive graphs with large vertex stabilizers, Discrete Math. 299 (2005), 180193.

[86] D. Marušič and R. Nedela, Maps and half-transitive graphs of valency 4, European J. Combin. 19 (1998), 345-354.

[87] D. Marušič and R. Nedela, On the point stabilizers of transitive groups with non-self-paired suborbits of length 2, J. Group Theory 4 (2001), 19-43.

[88] D. Marušič and R. Nedela, Finite graphs of valency 4 and girth 4 admitting half-transitive group actions, $J$. Austral. Math. Soc. 72 (2002), 1-16.

[89] D. Marušič and T. D. Parsons, Hamiltonian paths in vertex-symmetric graphs of order 5p, Discrete Math. 42 (1982), 227-242.

[90] D. Marušič and T. D. Parsons, Hamiltonian paths in vertex-symmetric graphs of order $4 p$, Discrete Math. 43 (1983), 91-96.

[91] D. Marušič and T. Pisanski, Weakly Flag-transitive Configurations and Half-arc-transitive Graphs, European J. Combin. 20 (1999), 559-570.

[92] D. Marušič and T. Pisanski, The GRAY graph revisited, J. Graph Theory 35 (2000) 1-7.

[93] D. Marušič, and T. Pisanski, Symmetries of Hexagonal Molecular Graphs, Croatica Chem. Acta 73 (2000) $969-981$.

[94] D. Marušič, T. Pisanski and S. Wilson, The genus of the Gray graph is 7, European J. Combin. 26 (2005), no. 3-4, 377-385.

[95] D. Marušič and P. Potočnik, Bridging semisymmetric and half-arc-transitive actions on graphs, European J. Combin. 23 (2002), 719732.

[96] D. Marušič and C. E. Praeger, Tetravalent graphs admitting half-transitive group actions: alternating cycles, J. Combin. Theory Ser. B 75 (1999), 188-205.

[97] D. Marušič and R. Scapellato, Permutation groups, vertex-transitive digraphs and semiregular automorphisms, European J. Combin. 19 (1998), 707-712. 
[98] D. Marušič and P. Šparl, On quartic half-arc-transitive metacirculants, J. Algebraic Combin., doi: 10.1007/s10801-007-0107-y, in press.

[99] D. Marušič and M. Y. Xu, A 1/2-transitive graph of valency 4 with a nonsolvable goup of automorphisms, $J$. Graph Theory 25 (1997), 133-138.

[100] Š. Miklavič, P. Potočnik and S. Wilson, Overlap in consistent cycles, J. Graph Theory 55 (2007), 55-71.

[101] Š. Miklavič, P. Potočnik and S. Wilson, Consistent cycles in half-arc-transitive graphs, preprint.

[102] T. Pisanski, A classification of cubic bicirculants, Discrete Math. 307 (2007), 567-578.

[103] T. Pisanski, Yet another look at the Gray graph, New Zealand J. Math. 36 (2007), 85-92.

[104] T. Pisanski, M. Boben, D. Marušič, A. Orbanić, A. Graovac, The 10-cages and derived configurations, Discrete Math. 275 (2004) 265 - 276.

[105] T. Pisanski, M. Randić, Bridges between Geometry and Graph Theory, in Geometry at Work, C.A. Gorini, ed., MAA Notes 53 (2000) $174-194$.

[106] C. E. Praeger, Imprimitive symmetric graphs, Ars Combinatoria 19A (1985), 149-163.

[107] C. E. Praeger, On automorphism groups of imprimitive symmetric graphs, Ars Combin. 23 (1987), $207-224$.

[108] C. E. Praeger, Finite normal edge-transitive Cayley graphs, Bull. Austral. Math. Soc. 60 (1999), 207-220.

[109] C. E. Praeger, Quotients and inclusions of finite quasiprimitive permutation groups, J. Algebra 269 (2003), 329-346.

[110] C. E. Praeger, Quasiprimitivity: structure and combinatorial applications, Discrete Math. 264 (2003), 211224.

[111] M. J. de Resmini and D. Jungnickel, Strongly regular semi-Cayley graphs, J. Algebr. Combin. 1 (1992), 171-195.

[112] M. Šajna, Half-transitivity of some metacirculants, Discrete Math. 185 (1998), 117-136.

[113] H. Schroeter, Über lineare Konstructionen zur Herstellung der Konfigurationen $n_{3}$, Nachr. Ges. Wiss. Göttingen (1888), 237-253.

[114] E. Steinitz, Konfigurationen der projektiven Geometrie, Encyclop. Math. Wiss. 3 (Geometrie) (1910), 481516.

[115] P. Šparl, A classification of tightly attached half-arc-transitive graphs of valency 4, J. Combin. Theory Ser. B, doi:10.1016/j.jctb.2008.01.001, in press.

[116] P. Šparl, On the classification of quartic half-arc-transitive metacirculants, Discrete Math., doi:10.1016/j.disc.2008.05.006, in press.

[117] D. E. Taylor and M. Y. Xu, Vertex-primitive 1/2-transitive graphs, J. Austral. Math. Soc. Ser. A 57 (1994), 113-124.

[118] C. Thomassen and M. E. Watkins, Infinite vertex-transitive, edge-transitive, non 1-transitive graphs, Proc. Amer. Math. Soc. 105 (1989), 258-261.

[119] J. Turner, Point-symmetric graphs with a prime number of points, J. Combin. Theory 3 (1967), 136-145.

[120] W. T. Tutte, A family of cubical graphs, Proc. Cambridge Philos. Soc. 43 (1947), 459-474.

[121] W. T. Tutte, Connectivity in graphs, University of Toronto Press, Toronto, 1966.

[122] C. Thomassen, Tilings of the torus and the Klein bottle and vertex-transitive graphs on a fixed surface, Trans. Amer. Math. Soc. 323 (1991), 605-635.

[123] R. J. Wang, Half-transitive graphs of order a product of two distinct primes, Comm. Algebra 22 (1994), 915-927.

[124] H. Wielandt, "Finite Permutation Groups”, Academic Press, New York 1964.

[125] H. Wielandt, "Permutation groups through invariant relations and invariant functions", Lecture Notes, Ohio State University, Columbus, 1969.

[126] R. Weiss, s-transitive graphs, Algebraic Methods in Graph Theory (Colloquia. Math. Soc. Janos Bolyai 25, North-Holland, Amsterdam, 1981), pp. 827-847.

[127] D. Witte, Cayley digraphs of prime-power order are Hamiltonian. J. Combin. Theory Ser. B 40 (1986), 107112. 
[128] J. Xu, Semiregular automorphisms of arc-transitive graphs with valency pq, European J. Combin., in print.

[129] M. Y. Xu, Half-transitive graphs of prime cube order, J. Algebraic Combin. 1 (1992), 275-282.

[130] S. Zhou, A local analysis of imprimitive symmetric graphs, J. Algebraic Combin. 22 (2005), 435-449. 